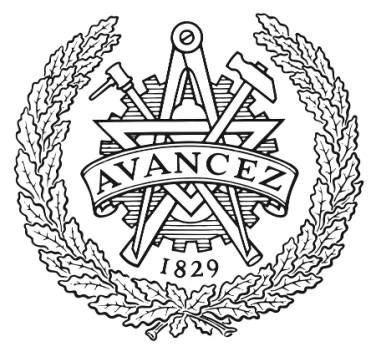

CHALMERS

UNIVERSITY OF TECHNOLOGY

\title{
Exploring Supramolecular Gels in Flow-Type Chemistry - Design and Preparation of Stationary Phases
}

Downloaded from: https://research.chalmers.se, 2023-04-26 13:03 UTC

Citation for the original published paper (version of record):

Zacharias, S., Kamlar, M., Sundén, H. (2021). Exploring Supramolecular Gels in Flow-Type Chemistry - Design and Preparation of Stationary

Phases. Industrial \& Engineering Chemistry Research, 60(28): 10056-10063.

http://dx.doi.org/10.1021/acs.iecr.1c01914

N.B. When citing this work, cite the original published paper. 


\title{
Exploring Supramolecular Gels in Flow-Type Chemistry-Design and Preparation of Stationary Phases
}

\author{
Savannah C. Zacharias, Martin Kamlar, and Henrik Sundén*
}

Cite This: Ind. Eng. Chem. Res. 2021, 60, 10056-10063

Read Online

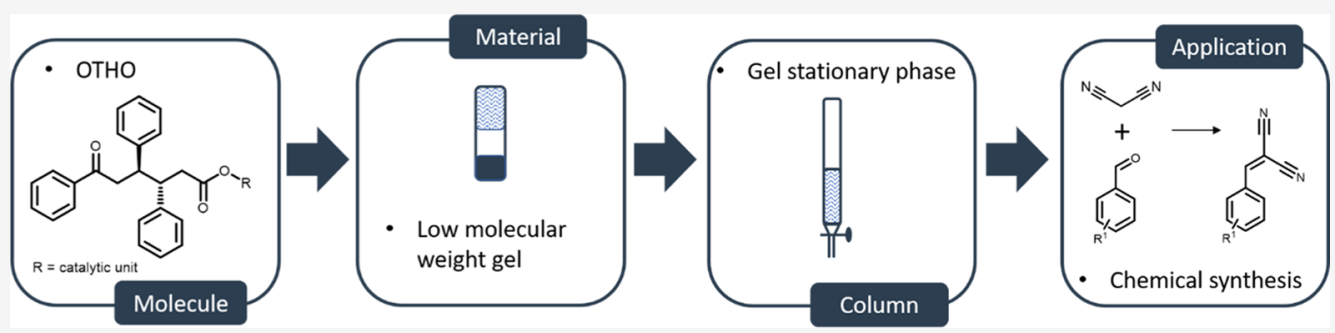

ABSTRACT: Two major challenges facing chemical synthesis are product isolation and catalyst recovery. One method to overcome these challenges is to perform the synthesis in a flow system with a catalytic stationary phase. However, the polymeric catalytic materials used in flow systems are often laborious to produce. In this study, we investigate a novel supramolecular gel as a catalytic stationary phase material. The gel is based on a modular, easy to synthesize, oxotriphenylhexanoate (OTHO) gelator comprised of a catalytic unit designed to catalyze the Knoevenagel reaction. The catalytic organogel enhances the rate of product formation and can be reused five times. Use of the OTHO to construct catalytic gels is a flexible technique that can be utilized to improve product isolation and reduce wastage of the catalyst.

\section{INTRODUCTION}

Much attention has been paid to the field of low-molecularweight gels (LMWGs) as these versatile materials are highly tunable and responsive to external stimuli. The self-assembly of supramolecular gels is driven by noncovalent interactions such as hydrogen bonding, van der Waals forces, and $\pi-\pi$ interactions. ${ }^{1}$ Consequently, this makes gels prime material candidates for various future applications such as drug delivery, $^{2}$ waste clean-up, ${ }^{3,4}$ energy storage, ${ }^{5}$ gas storage, sensing, ${ }^{6}$ and catalysis. ${ }^{7,8}$

One area of chemistry that has greatly benefitted from solidphase support is chemical synthesis. Solid supports are used in peptide synthesis ${ }^{9}$ as reagents ${ }^{10}$ in natural product synthesis $^{11-13}$ and for the immobilization of expensive metalbased catalysts. ${ }^{14}$ In this context, gels are being used to create custom support materials in the catalytic arena. There is a multitude of advantages when using gels for catalysis; these include high surface area, functional tunability, effective oxygen blocking (necessary for some reactions), directional and conformational orientation of the catalytic site, and slow diffusion through immobilized solvent-phase. ${ }^{15,16}$

The literature reflects the growing number of catalytic hydro-, ${ }^{17,18}$ organo-, ${ }^{19-24}$ and organometallic- ${ }^{8,25-32}$ gels. Gels have been used in a wide range of catalytic reactions including Heck, ${ }^{31}$ Diels-Alder, ${ }^{29}$ Aldol, $^{22-24,30}$ Suzuki-Miyaura coupling, ${ }^{31} \mathrm{~N}$-alkylation, ${ }^{32}$ and photochemical reactions. ${ }^{15}$ Hydroand organogels differ in the nature of the liquid immobilized; this is either organic solvent (organogels) or water (hydro- gels). Most organic syntheses are performed in organic solvents. This makes catalytic organogels enticing as they can gelate in a broad range of organic solvents, making them applicable for a wider range of chemical reactions than hydrogels.

Two strategies have been employed to create catalytic gels either by encapsulation of the catalyst by the $\mathrm{gel}^{33}$ or through synthetic modification of the gelator. In the first method, the catalyst is not covalently bound to the gelator and is free to diffuse through the gel pockets. In the second method, the catalytic unit is covalently bonded to the gelator. Synthesis of a catalytic gelator is often laborious and complex as it is difficult to synthesize a catalyst and guarantee gelation ability. ${ }^{34} \mathrm{Few}$ examples are reflected in the literature for the approach where the catalytic unit is bound to the gelator. ${ }^{23,24,32}$ Furthermore, many catalytic reactions using catalytic gels are studied in situ and followed using techniques such as $\mathrm{NMR}^{22}$ and HPLC. ${ }^{23}$ In the cases where the product is isolated, the gel is often destroyed making it unsuitable for reuse. ${ }^{32} \mathrm{~A}$ solution to overcome these obstacles would be to use supramolecular gels

Received: May 19, 2021

Revised: June 25, 2021

Accepted: June 25, 2021

Published: July 9, 2021
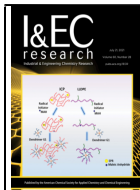


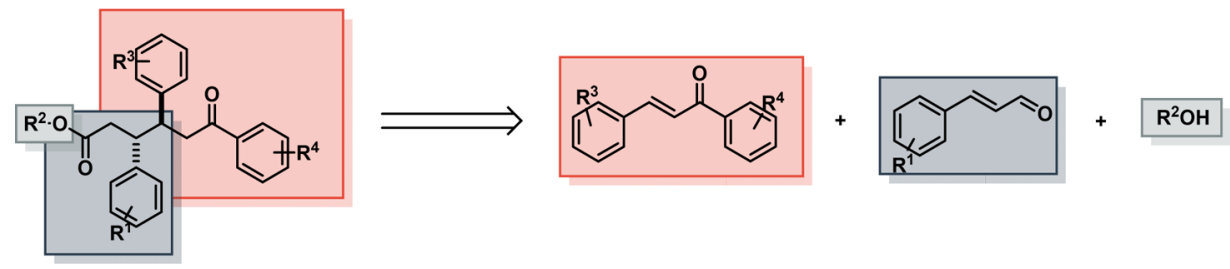

Figure 1. Highly modular OTHO reaction allows for the design and synthesis of a vast array of gelators.

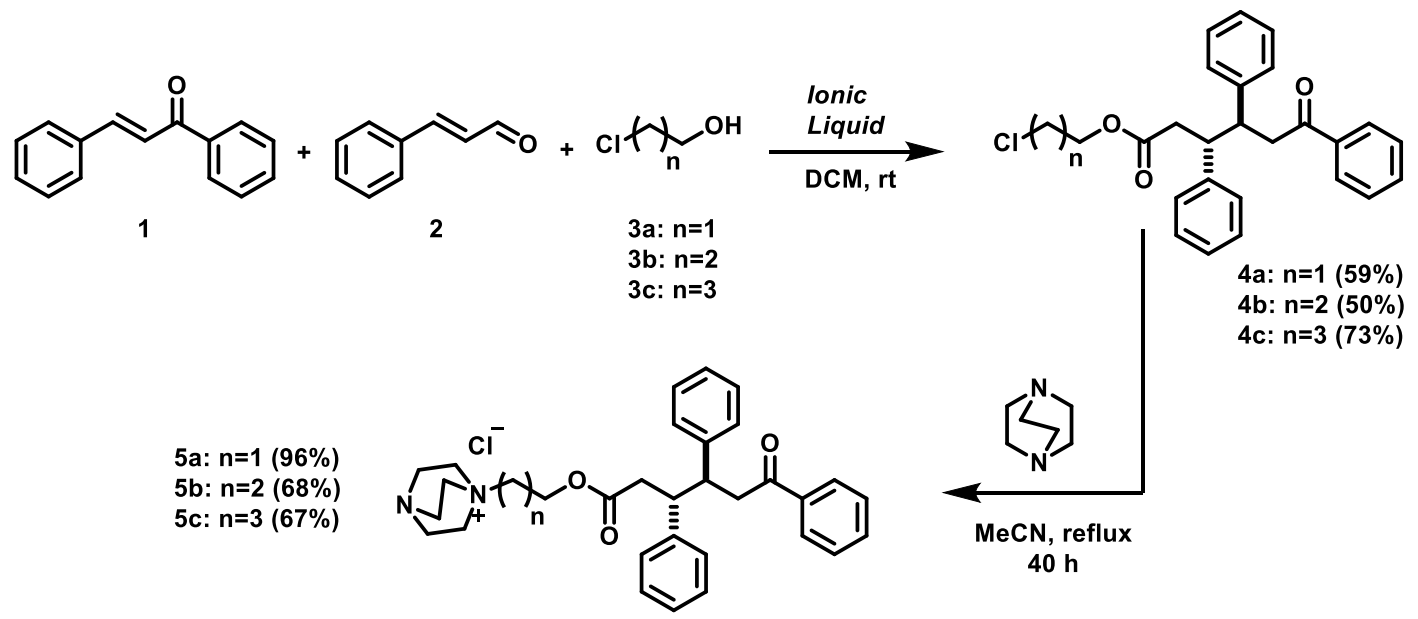

Figure 2. Two-step synthetic pathway for the synthesis of three different OTHO-DABCO compounds (5a, $\mathbf{5 b}$, and $\mathbf{5} \mathbf{c})$ with different length linkers.

in a flow-type system. ${ }^{18,35}$ Flow-type chemistry provides an easy, swift, clean, and efficient separation of the product and the catalyst, as well as a method for catalyst recovery.

It is therefore desirable to create a synthetically flexible gelator that could be assembled in a limited number of steps to enable efficient screening of both gel and catalytic properties.

Oxotriphenylhexanoate $(\mathrm{OTHO})^{36-40}$ is a prime candidate for the development of a catalytic organogel. The synthesis of OTHO is straightforward using readily available starting materials; the compound is easy to purify, and the reaction scales efficiently. In addition, OTHO is synthetically flexible, and the OTHO backbone can be modified with a variety of functional groups that can be used to design a gelator for a desired purpose (Figure 1).

It is advantageous to have a system for catalysis which allows for easy separation of the product and the catalyst, reuse of the catalyst and is compatible with many solvents. It is an added benefit if the synthesis of this catalyst is straightforward. In this work, we design and synthesize a novel catalytic gelator for use in a flow-type system allowing for high reaction efficiency, easy product isolation, and reuse of the gel catalyst.

\section{RESULTS AND DISCUSSION}

In order to explore supramolecular gels as a flow-type stationary phase, two different approaches were used. The first involves encapsulating a catalyst in the gel pockets. The second method utilizes synthetic modification of OTHO to create a gelator capable of catalysis. Tertiary amines were identified as the catalytic units of choice as they are known to catalyze a number of reactions ${ }^{41-44}$ that are operationally simple and can be performed at room temperature. The first method was unsuccessful as the catalyst coeluted with the product, making the material unsuitable for reuse (details in Supporting Information). To address this, the catalytic unit 1,4-diazabicyclo[2.2.2] octane (DABCO) was covalently bound to the OTHO.

\section{BUILDING A SUPPORT FOR CATALYSIS}

Synthesis of Novel Catalytic Gelator. During the design process, it was decided to attach the DABCO to the ester part of the OTHO via a carbon linker. We postulated that the linker might influence gelation and the catalytic properties of the gel. Thus, three different OTHOs were synthesized, each with a different length carbon linker. The linker lengths were 2carbon, 3-carbon, and 4 -carbon for $\mathbf{5 a}, \mathbf{5 b}$, and $\mathbf{5 c}$, respectively (Figure 2).

The two-step gelator synthesis starts with an NHC-catalyzed three-component reaction ${ }^{36,37}$ for the synthesis of alkyl halogen OTHOs $\mathbf{4 a - c}$ (Figure 2). trans-Chalcone, cinnamaldehyde, and appropriate alcohol were reacted at room temperature in dichloromethane (DCM) in the presence of 1-ethyl-3-methylimidazolium acetate and 1,8diazabicyclo[5.4.0] undec-7-ene (DBU). The precursor OTHOs were obtained in a pure form as a solid material following a simple precipitation and washing protocol using methanol.

In the final step, the alkyl halogen containing OTHOs (4ac) were treated with $\mathrm{DABCO}$ under refluxing conditions in acetonitrile $(\mathrm{MeCN})$. Following a filtration and washing procedure, the catalytic OTHO-DABCOs $(\mathbf{5 a}-\mathbf{c})$ were obtained in good yields. Procedure details are described in the Supporting Information.

Gelation of Catalytic OTHO-DABCOs 5a, 5b, and 5c. There are multiple methods for triggering gelation. ${ }^{45-49} \mathrm{We}$ investigated cooling and sonication and found that sonication was the optimal method for triggering gelation. Sonication is a known method for triggering gelation, ${ }^{48,49}$ and a number of explanations for this behavior have been presented. ${ }^{50-55}$ 


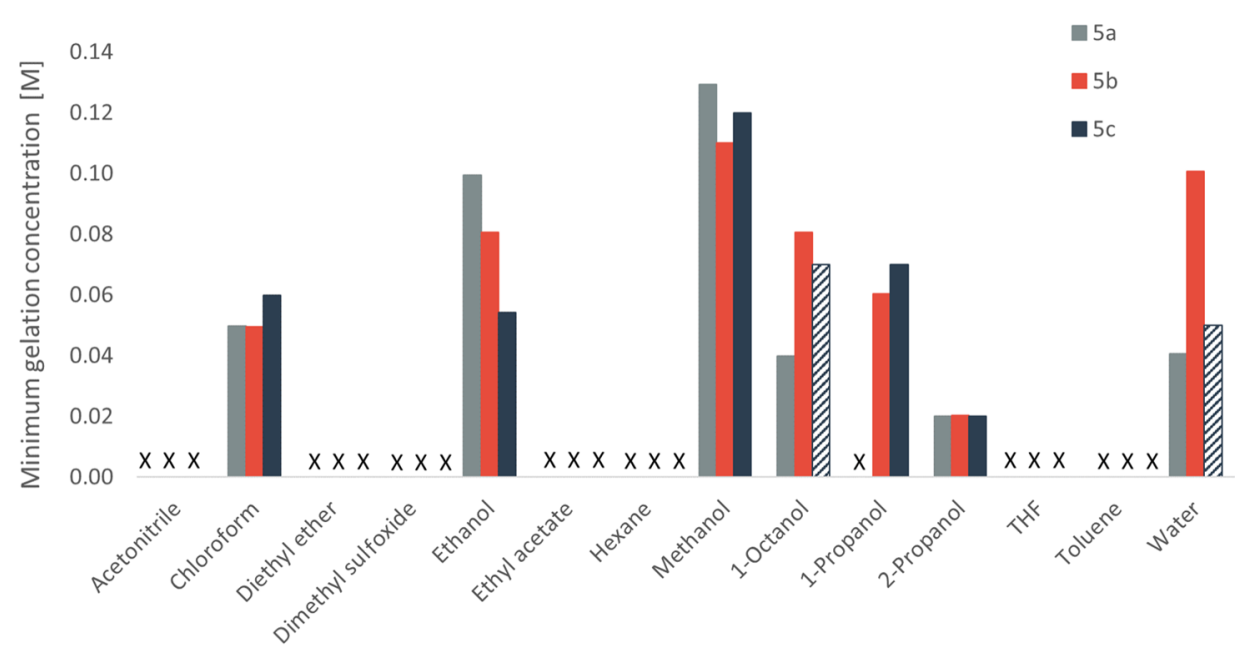

Solvent

Figure 3. Minimum gelation concentration for each OTHO-DABCO (5a, $\mathbf{5 b}$, and $\mathbf{5 c})$. The length of the carbon linker influences the gelation. X indicates that gel was not formed. The striped bars represent unstable gels.

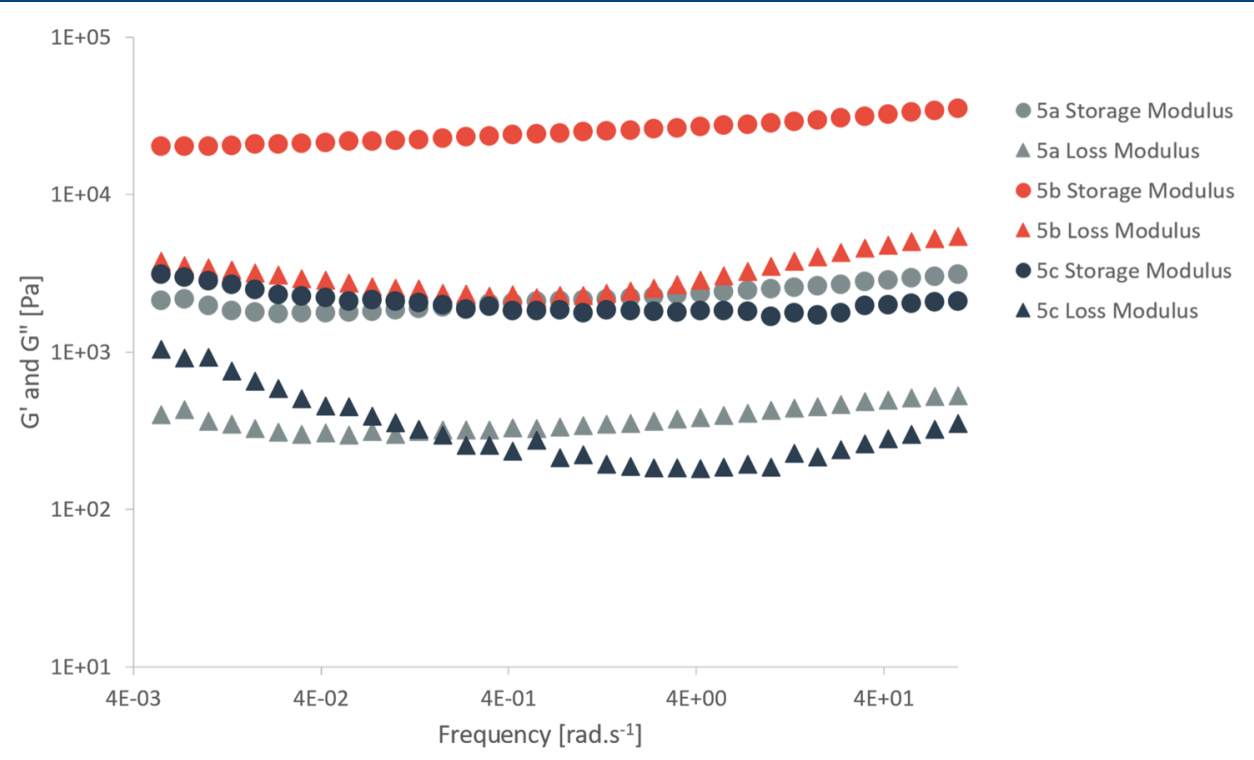

Figure 4. Storage (circle) and loss (triangle) moduli of $\mathbf{5 a}, \mathbf{5 b}$, and $\mathbf{5} \mathbf{c}$ as a function of frequency. The frequency sweep was performed at a strain of $0.1 \%$ and $T=25{ }^{\circ} \mathrm{C}$. All of the gels are made from $i \mathrm{PrOH}$ and have a concentration of $2 \times 10^{-2} \mathrm{M}$.

Gelation Study. To test the range of solvents compatible for gelation and to obtain the minimum gelation concentration (MGC) in each solvent, a gelation study was performed. The compound (5a, $\mathbf{5 b}$, or $\mathbf{5 c}$ ), starting with an amount of $1 \times 10^{-5}$ mol, was dissolved in a solvent $(1 \mathrm{~mL})$ in a sealed vial using a heat-gun. This solution was sonicated in a benchtop sonicator until either a gel or a precipitate was formed. The temperature of the sonicator water bath was kept between 18 and $23{ }^{\circ} \mathrm{C}$. If neither gelation nor precipitation occurred, OTHO-DABCO compound (5a, $\mathbf{5 b}$, or $\mathbf{5 c}$ ) was added in $1 \times 10^{-5}$ mol portions until gelation or precipitation occurred. The inverted vial test was used to identify gel formation. ${ }^{56}$

The linker length impacts the properties of the compounds, and the three compounds, $\mathbf{5 a}, \mathbf{5 b}$, and $\mathbf{5 c}$, behave differently. This is clearly demonstrated by the gelation ability of the series (Figure 3 and Supporting Information Table S2).

During the solvent screening, it was found that $5 \mathbf{a}-\mathbf{c}$ form gels in organic solvents such as chloroform and various alcohols. All three compounds were insoluble in diethyl ether, hexane, and toluene. Precipitate was formed in $\mathrm{MeCN}$ and tetrahydrofuran.

All three gelators form hydrogels at varying concentrations. Although 5c does form a hydrogel, it was unstable and the gel degraded within $30 \mathrm{~min}$. Organic solvent is more useful for organic synthesis, and therefore no further investigation into the hydrogels were performed.

The MGC of 5a was the most variable, and 5c forms the least stable gels. The greatest number of stable gels was formed by $\mathbf{5 b}$ indicating that the 3 -carbon length linker $(\mathbf{5 b})$ is the optimal length for gelation. The 3-carbon length linker provides the correct lipophilicity resulting in the desired balance between solubility and insolubility for gel formation. ${ }^{57}$

The most appropriate solvent for comparing the catalytic ability of the gelators is $i \mathrm{PrOH}$ since all gelators have the same MGC $\left(2 \times 10^{-2} \mathrm{M}\right)$, and the resulting gels are stable. Thus, $i \mathrm{PrOH}$ makes it possible to compare the catalytic ability of the gelators at the same concentration. In order to explore the physical properties of the gels and examine how this may 
impact catalysis, characterization of the $i \mathrm{PrOH}$ gels was performed.

Rheology. Dynamic shear oscillation was used to investigate the rheological properties of the gels. Storage modulus $\left(G^{\prime}\right)$ and loss modulus $\left(G^{\prime \prime}\right)$ were measured as a function of frequency and strain. For all three gels, $G^{\prime}$ is 1 order of magnitude larger than $G^{\prime \prime}$ (Figure 4); this is seen in tangled fibrillar networks such as cellulose nanofibrils ${ }^{58}$ and other LMWGs. ${ }^{59}$ The differences in the viscosities can be explained by the differences in the strengths of the interactions within the gel network.

The softest gels are 5a and 5c. The softness of $\mathbf{5 a}$ may be attributed to the steric hindrance and electrostatics in combination with the solvent used $(i \mathrm{PrOH})$. The steric hindrance is a result of the large, bulky, charged amino groups positioned in such a way (without the freedom of movement the 3-carbon and 4-carbon linkers provide) that they repel each other. The 2-carbon linker does not allow for rotational movement to relieve this strain. Gelator $\mathbf{5 c}$ has a longer, more flexible 4-carbon linker than $\mathbf{5 b}$, and this may lead to greater conformational freedom of the DABCO functional group, so the network is more flexible, leading to a lower shear viscosity. A similar trend is seen with modified cellulose nanocrystals. ${ }^{60}$ The 3-carbon linker of $\mathbf{5 b}$ appears to provide a balance between the steric hindrance caused by the bulky DABCO group and flexibility and freedom of rotation that results from a longer carbon chain. For this system, a 3-carbon linker is the optimum length to allow for stronger intermolecular forces in $i \mathrm{PrOH}$.

Care must be taken when handling the samples before measurement ${ }^{61,62}$ as the gels are often not viscous enough to remain intact during transfer from the sonication vessel to the plate. Because of this, it is difficult to get an exact quantification of the storage and loss moduli. However, the trends observed are reproducible.

Morphology of iPrOH Gels. The different linkers result in visually different gels (Figure 5a). Although on a macroscopic scale, the gels were different, SEM images revealed flat, ribbonlike structures in xerogels for all three in $i \mathrm{PrOH}$ (Figure $5 b-d)$. The least uniform in terms of fiber size, ranging from 0.2 to $1.0 \mu \mathrm{m}$, was $\mathbf{5 a}$. The fibers for $\mathbf{5 b}$ had a size range of 0.3 a)

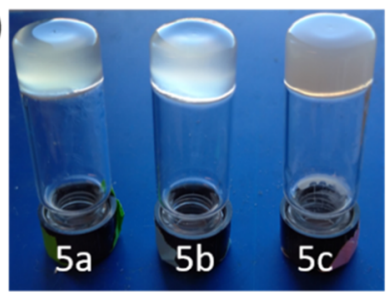

c)

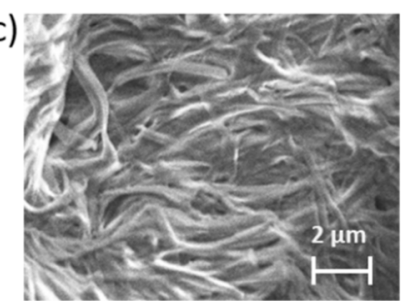

b)

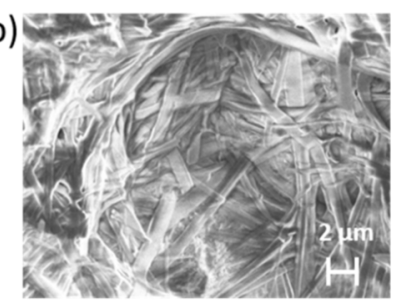

d)

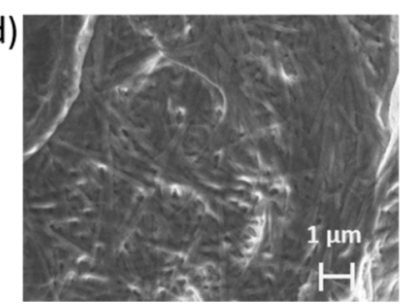

Figure 5. (a) Gels in iPrOH 5a, 5b, and 5c (left to right). The gels are visibly different. The compound with the longest linker, $\mathbf{5 c}$, is the opaquest. SEM images of (b) 5 a, (c) 5 b, and (d) 5 c. All three gels have similar morphologies (all fibers). to $0.6 \mu \mathrm{m}$. 5c had the least broad, most uniform fibers $(0.3$ $\mu \mathrm{m})$. All gels exhibited fiberlike morphologies indicating that the solvent did not have an effect on the morphology (Supporting Information Figure S4).

Powder X-ray Diffraction. The powder X-ray diffraction patterns show that the xerogels are semicrystalline, and there are differences in the packing of the molecules in the solid phase (Supporting Information Figure S5). The differences in packing may lead to different arrangements of the active site and hence the catalytic ability of the material.

Catalysis Using Catalytic Gel in Flow-Type System. To evaluate the gelator catalytic performance as a stationary phase, we chose the Knoevenagel reaction as it is known to be catalyzed by tertiary amines. $^{63,64}$

Control reactions performed in solution required $3 \mathrm{~h}$ to reach full conversion. The reaction rate is notably improved when the gel phase was used, and full conversion was reached within an hour (Supporting Information Table S4).

Preparation of Gel Column for Catalysis. The OTHODABCO gelator was dissolved in $\mathrm{PrOH}\left(4 \mathrm{~mL}, 2 \times 10^{-2} \mathrm{M}\right)$ with heating, and the warm solution was poured into a glass column packed with approximately $40 \mathrm{mg}$ of cotton wool. Gelation was triggered via sonication in the column (Supporting Information Figure S7a) using a benchtop sonicator. The resulting gel was washed with $i \mathrm{PrOH}(8 \mathrm{~mL})$ to remove ungelated material from the gel pockets prior to substrate loading. A solution of the substrate was gently added on top of the gel. The tap was closed, and the column stoppered for $60 \mathrm{~min}$. The substrates diffuse through the gel pockets $^{39}$ where they interact with the catalytic sites and the reaction proceeds. The column was then eluted using $100 \mathrm{~mL}$ of EtOAc (Supporting Information Figure S7b). The remaining gel was dissolved in DCM for further analysis by NMR.

The use of a glass column has several advantages including, straightforward removal of the ungelated compound before catalysis with washing and simple separation of the product from the gel phase. In addition, the use of a transparent glass column provides the ability to monitor the reaction visually and detect precipitation and changes in the morphology and color.

Catalysis. The catalytic ability of the three gelators, $\mathbf{5 a - c}$ were compared (Table 1). For all experiments, the reaction proceeds with quantitative conversion of the starting material. Gelator 5a was able to catalyze the reaction between benzaldehyde and malononitrile with $100 \%$ conversion and crude yield of $94 \%$ (Table 1, entry 1). However, a large amount of 5a was washed out of the gel column during the elution process, indicating that this gel is not very stable. As seen during the gelation study, $\mathbf{5 c}$ was the least stable gel and the gel degrades during the elution of the product (Table 1, entry 3 ). Gelator $\mathbf{5 b}$ was visibly the most stable gel, and only a small amount of the gelator was present in the product mixture (Table 1, entry 2). Despite having the lowest crude yield, this stability makes this system more attractive than the other two. From the rheological results, $\mathbf{5 b}$ was the strongest gel (Figure 4 and Supporting Information Figure S2-S3). This is a result of the optimal chain length and consequently stronger intermolecular forces within the gel network. Therefore, gelator $\mathbf{5 b}$ was used for further investigation because it has the desirable physical properties, the best isolation of product, and $100 \%$ conversion. Irrespective of the system, analysis of 
Table 1. Comparison of Knoevenagel Reaction between Benzaldehyde and Malononitrile Using iPrOH Gels of 5a, $5 b$, and 5c ${ }^{a}$

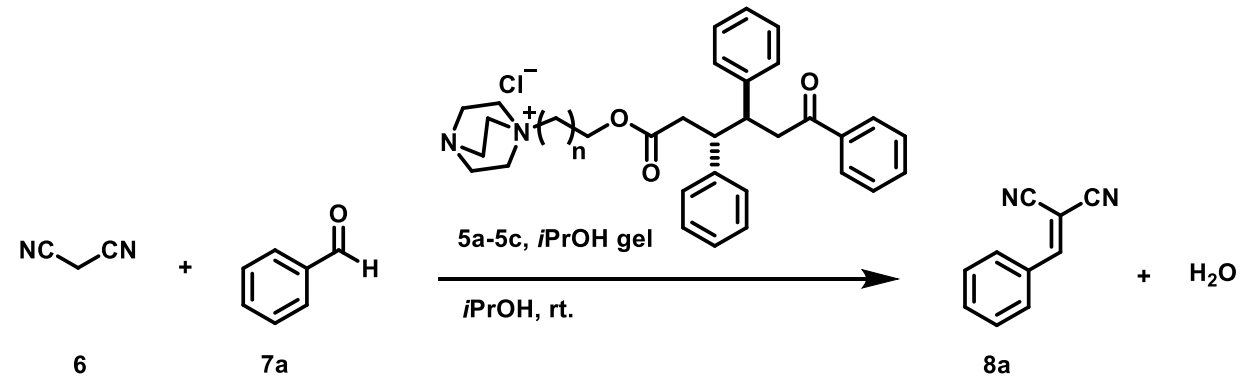

\begin{tabular}{|c|c|c|c|c|c|}
\hline entry & catalyst & conversion $[\%]$ & crude yield $[\%]^{b}$ & coeluted gelator $[\%]^{c}$ & product encapsulated in gel $[\%]^{d}$ \\
\hline 1 & $\mathbf{5 a}(n=1)$ & 100 & 94 & 15 & 22 \\
\hline 2 & $\mathbf{5 b}(n=2)$ & 100 & 69 & 6 & 35 \\
\hline 3 & 5c $(n=3)$ & 100 & 83 & 13 & 45 \\
\hline
\end{tabular}

${ }^{a}$ Full conversion was reached within $1 \mathrm{~h}$ in each instance, and $i \operatorname{PrOH}(100 \mathrm{~mL})$ was used for elution. ${ }^{b}$ Calculated using the mass of eluted material after drying. ${ }^{c}$ Percentage of $\mathbf{5}$ coeluted with the product, based on NMR data. ${ }^{d}$ Percentage of product trapped in gel portion after elution. Calculated using NMR data collected on the dissolved gel after elution.

Table 2. Study Into the Reuse of the Gel-5b Was Used Five Times without Significant Loss of the Gelator ${ }^{a}$

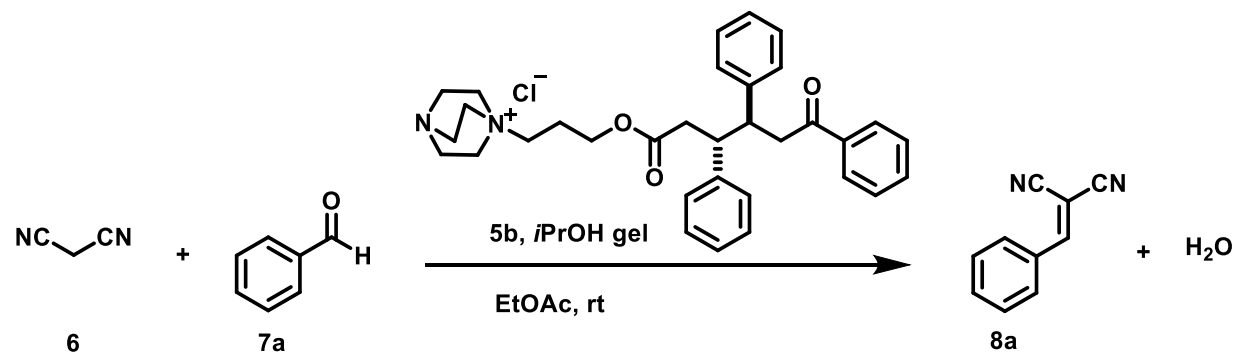

$\begin{array}{cccc}\text { run } & \text { conversion }[\%] & \text { crude yield }[\%]^{b} & {\text { coeluted gelator }[\%]^{c}}^{c} \\ 1 & 100 & 90 & 9 \\ 2 & 100 & 77 & 47 \\ 3 & 100 & 63 & \text { trace } \\ 4 & 92 & 36 & \text { trace } \\ 5 & 80 & 43 & \text { trace }\end{array}$

${ }^{a}$ Reactions were carried out on a gel of $\mathbf{5 b}(0.8 \mathrm{mmol})$ from $i \mathrm{PrOH}$ at room temperature; the starting materials, $\mathbf{6}(0.3 \mathrm{mmol})$ and $7 \mathbf{a}(0.45 \mathrm{mmol})$, were loaded as an EtOAc $(1 \mathrm{~mL})$ solution. ${ }^{b}$ Calculated using the mass of eluted material after drying. ${ }^{c}$ Percentage of $\mathbf{5 b}$ coeluted with the product, based on NMR data.

the gel after product isolation with NMR reveals that some product always remains trapped in the gel phase.

After testing various solvents, it was found that using EtOAc $(100 \mathrm{~mL})$ instead of $i \mathrm{PrOH}$ resulted in faster elution time, greater product isolation, and less dissolution of the $\mathbf{5 b}$ gel (Supporting Information Figure S8, Table S5-S6).

The number of times the gel could be reused was tested, and at least five successive reactions could be performed on the same gel without large amounts of $\mathbf{5} \mathbf{b}$ washing out (Table 2 ). There is a sharp decrease in yield after the fourth reaction, and this performance is comparable with the other gel catalyst. ${ }^{25}$ The trend in the yield can be explained by the amount of product that remains trapped within the gel. After three consecutive uses, the concentration of the product within the gel pockets is high, and as a result access to the catalytic units is blocked. The slight improvement in the yield for the fifth run $(36-43 \%)$ is most likely due to the product from the previous reactions being eluted. Full conversion is not reached for the fifth reaction. The remaining gel phase is dissolved after the last run, and ${ }^{1} \mathrm{H} \mathrm{NMR}$ revealed that the gel phase was made up of $\sim 50 \%$ product.
There are several reasons for the loss in activity including the hindrance of access of the catalytic site. These blockages can be caused by the incomplete removal of the product from the active site, the partial collapse of the gel pocket, or the formation of a stable ion pair between the ammonium ion and the anion of the malonitrile.

To investigate the impact of the gel phase on the catalytic activity, $\mathbf{5} \mathbf{b}$ gel was replaced by $\mathbf{5 b}$ in the solid phase (as a precipitate from EtOAc) and catalysis was carried out in an identical fashion. In this case, only 55\% conversion to 8 a was achieved, clearly demonstrating the importance of the gel pocket in efficient catalysis, which allows the substrates to flow through the material and interact with the catalytic sites. In addition, it provides a larger surface area for the reaction.

Examining the gels before and after use via SEM revealed changes to the material. After the catalytic reaction, the fibers shrink and solids could be seen entwined in the fibers (Figure 6). These solids block the catalytic sites and result in lower yields during reuse. NMR analysis of the gel after catalysis showed that only the product and OTHO-DABCO were present, confirming that the large, white structures in the SEM micrographs are the product. 

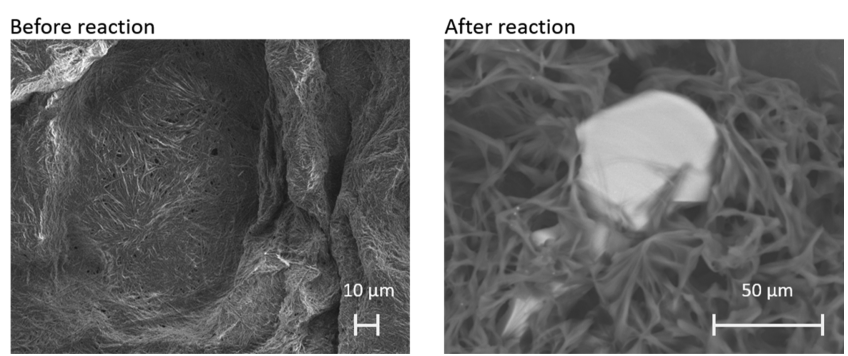

Figure 6. Gel network of $\mathbf{5 b}$ before and after use. Both SEM micrographs have a magnification of $1.45 \mathrm{k} \times$. The precipitated product can be seen entwined in the network. This product blocks catalytic sites and the reaction cannot proceed, resulting in lower yields. NMR shows that only the gelator and the product are present.

Substrate Scope. In order to test the functional group tolerance of the catalytic system as well as how compatible the gel is with different functional groups, the substrate scope was investigated. All reactions performed well with yields ranging from 65 to $69 \%$ (Figure 7). For example, halogen substituents are well tolerated and $p$-chloro substituted benzaldehydes can be used as substrates in the reaction affording the corresponding product $(\mathbf{8 b})$ in $68 \%$ yield, revealing that the system is broadly compatible with a variety of substrates, making this a robust material for the Knoevenagel reaction.

In general, the gel network is retained during the reaction for most functional groups present in the benzaldehyde starting material. However, $p$-nitro benzaldehyde appears to disrupt the gel network. As a result, an increased amount of the gelator was washed out during elution, and the product was trapped in the gel $(45 \%)$ to a greater extent than the other substrates (between 15 and 25\%).

\section{CONCLUSIONS}

Catalytic supramolecular gels provide an elegant solution for the challenges of separation and catalytic species recovery and reuse. In this study, we show that a catalytic gelator can be designed and synthesized with desirable physical properties for our technique. The OTHO gelator can be converted into a catalytic gel that is operational under flow-type conditions. The catalytic system can be reused at least five times and is compatible with a number of substrates. We were also able to show that the major contributor to the loss of catalytic activity is accumulation of product in the gel.

We believe that supramolecular gels have the potential to pave the way for new developments of custom catalytic stationary phases as they can be readily designed and prepared from low-molecular-weight gelators. Currently, our group is investigating the challenges associated with robustness, solvent compatibility, and sustained catalytic efficiency in catalysis.

\section{ASSOCIATED CONTENT}

\section{SI Supporting Information}

The Supporting Information is available free of charge at https://pubs.acs.org/doi/10.1021/acs.iecr.1c01914.

General procedure for OTHOs 4a-c preparation; general procedure for OTHO-DABCO synthesis; gelation study; characterization of gels; experimental setup; optimization of conditions for catalysis; and NMR spectra of synthesized OTHOs (PDF)

\section{AUTHOR INFORMATION}

\section{Corresponding Author}

Henrik Sundén - Department of Chemistry and Molecular Biology, University of Gothenburg, SE-412 96 Gothenburg, Sweden; Department of Chemistry and Chemical Engineering, Chalmers University of Technology, 41296 Gothenburg, Sweden; 10 orcid.org/0000-0001-6202-7557; Email: henrik.sunden@chem.gu.se

\section{Authors}

Savannah C. Zacharias - Department of Chemistry and Molecular Biology, University of Gothenburg, SE-412 96 Gothenburg, Sweden; 1 orcid.org/0000-0003-0895-9120

Martin Kamlar - Department of Chemistry and Chemical Engineering, Chalmers University of Technology, 41296 Gothenburg, Sweden; Present Address: Department of Organic Chemistry, Faculty of Science, Charles University, Hlavova 2030/8, 12843 Prague 2, Czech Republic

Complete contact information is available at: https://pubs.acs.org/10.1021/acs.iecr.1c01914

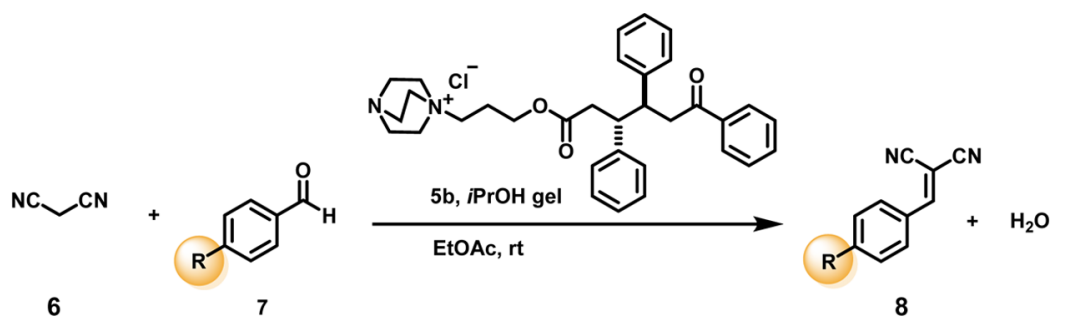

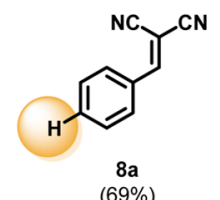

$(69 \%)$

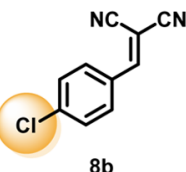

$(68 \%)$

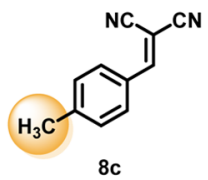

$(65 \%)$

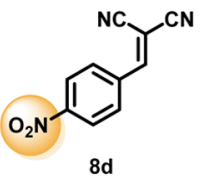

$8 d$
$(65 \%)$

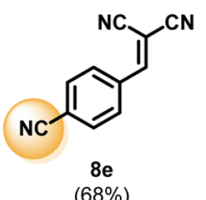

$(68 \%)$

Figure 7. Substrate scope. All reactions reached full conversion within $1 \mathrm{~h}$ in the gel. $100 \mathrm{~mL}$ of EtOAc was used to elute the product. Isolated yields are given. Reactions were carried out on an $i \mathrm{PrOH}$ gel of $\mathbf{5 b}(0.8 \mathrm{mmol})$ at room temperature; substrates $6(0.3 \mathrm{mmol}, 1$ equiv) and 7 (0.45 mmol, 1.5 equiv) were loaded as an EtOAc $(1 \mathrm{~mL})$ solution. 


\section{Author Contributions}

The manuscript was written through contributions of all authors. All authors have given approval to the final version of the manuscript.

\section{Notes}

The authors declare no competing financial interest.

\section{ACKNOWLEDGMENTS}

This work was supported by grants from the Swedish research council FORMAS (2019-00699) and from Olle Engkvists Stiftelse. S.C.Z. would like to thank Olle Engkvist Stiftelse and the A-forsk Foundation for the postdoctoral fellowship funding. M.K. thanks the Operational Programme Research, Development and Education (Project Improvement of internationalization in the field of research and development) at Charles University through the support of quality projects MSCA-IF (CZ.02.2.69/0.0/0.0/17_050/ 0008466) for postdoctoral funding. This work was performed in part at the Chalmers Material Analysis Laboratory, CMAL. Rheology data were, in part, collected with the kind assistance of the Roland Kádár research group.

\section{ABBREVIATIONS}

$\begin{array}{ll}\text { DCM } & \text { dichloromethane } \\ \text { EtOAc } & \text { ethyl acetate } \\ \text { iPrOH } & \text { 2-propanol } \\ \text { PXRD } & \text { powder X-ray diffraction } \\ \text { MGC } & \text { minimum gelation concentration } \\ \text { OTHO } & \text { oxotriphenylhexanoate } \\ \text { THF } & \text { tetrahydrofuran }\end{array}$

\section{REFERENCES}

(1) Buerkle, L. E.; Rowan, S. J. Supramolecular Gels Formed from Multi-Component Low Molecular Weight Species. Chem. Soc. Rev. 2012, 41, 6089-6102.

(2) Esposito, C. L.; Kirilov, P.; Roullin, V. G. Organogels, Promising Drug Delivery Systems: An Update of State-of-the-Art and Recent Applications. J. Controlled Release 2018, 271, 1-20.

(3) Rizzo, C.; Andrews, J. L.; Steed, J. W.; D’Anna, F. CarbohydrateSupramolecular Gels: Adsorbents for Chromium(VI) Removal from Wastewater. J. Colloid Interface Sci. 2019, 548, 184-196.

(4) Okesola, B. O.; Smith, D. K. Applying low-molecular weight supramolecular gelators in an environmental setting - self-assembled gels as smart materials for pollutant removal. Chem. Soc. Rev. 2016, 45, 4226-4251.

(5) Bera, S.; Haldar, D. A Rechargeable Self-Healing Safety Fuel Gel. J. Mater. Chem. A 2016, 4, 6933-6939.

(6) Sengupta, S.; Mondal, R. A Novel Low Molecular Weight Supergelator Showing an Excellent Gas Adsorption, Dye Adsorption, Self-Sustaining and Chemosensing Properties in the Gel State. RSC Adv. 2016, 6, 14009-14015.

(7) Harris, R. F.; Nation, A. J.; Copeland, G. T.; Miller, S. J. A Polymeric and Fluorescent Gel for Combinatorial Screening of Catalysts. J. Am. Chem. Soc. 2000, 122, 11270-11271.

(8) Xing, B.; Choi, M.-F.; Xu, B. Design of Coordination Polymer Gels as Stable Catalytic Systems. Chem.-Eur. J. 2002, 8, 5028-5032.

(9) Bodé, C. A.; Muller, C. P.; Madder, A. Validation of a SolidPhase-Bound Steroid Scaffold for the Synthesis of Novel Cyclic Peptidosteroids. J. Pept. Sci. 2007, 13, 702-708.

(10) Ley, S. V.; Baxendale, I. R.; Bream, R. N.; Jackson, P. S.; Leach, A. G.; Longbottom, D. A.; Nesi, M.; Scott, J. S.; Storer, R. I.; Taylor, S. J. Multi-Step Organic Synthesis Using Solid-Supported Reagents and Scavengers: A New Paradigm in Chemical Library Generation. J. Chem. Soc. Perkin Trans. 1 2000, 3815-4195.
(11) Eifler-Lima, V. L.; Graebin, C. S.; Uchoa, F. D. T.; Duarte, P. D.; Corrêa, A. G. Highlights in the Solid-Phase Organic Synthesis of Natural Products and Analogues. J. Braz. Chem. Soc. 2010, 21, 14011423.

(12) Gu, W.; Silverman, R. B. Solid-Phase Total Synthesis of Scytalidamide A. J. Org. Chem. 2003, 68, 8774-8779.

(13) Hall, D. G.; Manku, S.; Wang, F. Solution- and Solid-Phase Strategies for the Design, Synthesis, and Screening of Libraries Based on Natural Product Templates: A Comprehensive Survey. J. Comb. Chem. 2001, 3, 125-150.

(14) Ndolomingo, M. J.; Bingwa, N.; Meijboom, R. Review of Supported Metal Nanoparticles: Synthesis Methodologies, Advantages and Application as Catalysts. J. Mater. Sci. 2020, 55, 6195-6241.

(15) Maiti, B.; Abramov, A.; Pérez-Ruiz, R.; Díaz Díaz, D. The Prospect of Photochemical Reactions in Confined Gel Media. Acc. Chem. Res. 2019, 52, 1865-1876.

(16) Dawn, A. Supramolecular Gel as the Template for Catalysis, Inorganic Superstructure, and Pharmaceutical Crystallization. Int. J. Mol. Sci. 2019, 20, 781-803.

(17) Zozulia, O.; Dolan, M. A.; Korendovych, I. V. Catalytic Peptide Assemblies. Chem. Soc. Rev. 2018, 47, 3621-3639.

(18) Rodon Fores, J.; Criado-Gonzalez, M.; Chaumont, A.; Carvalho, A.; Blanck, C.; Schmutz, M.; Serra, C. A.; Boulmedais, F.; Schaaf, P.; Jierry, L. Supported Catalytically Active Supramolecular Hydrogels for Continuous Flow Chemistry. Angew. Chem., Int. Ed. 2019, 58, 18817-18822.

(19) Rodríguez-Llansola, F.; Escuder, B.; Miravet, J. F. Remarkable Increase in Basicity Associated with Supramolecular Gelation. Org. Biomol. Chem. 2009, 7, 3091.

(20) Mazzier, D.; Carraro, F.; Crisma, M.; Rancan, M.; Toniolo, C.; Moretto, A. A Terminally Protected Dipeptide: From Crystal Structure and Self-Assembly, through Co-Assembly with CarbonBased Materials, to a Ternary Catalyst for Reduction Chemistry in Water. Soft Matter 2016, 12, 238-245.

(21) Tobin, J. M.; McCabe, T. J. D.; Prentice, A. W.; Holzer, S.; Lloyd, G. O.; Paterson, M. J.; Arrighi, V.; Cormack, P. A. G.; Vilela, F. Polymer-Supported Photosensitizers for Oxidative Organic Transformations in Flow and under Visible Light Irradiation. ACS Catal. 2017, 7, 4602-4612.

(22) Singh, N.; Zhang, K.; Angulo-Pachón, C. A.; Mendes, E.; Van Esch, J. H.; Escuder, B. Tandem Reactions in Self-Sorted Catalytic Molecular Hydrogels. Chem. Sci. 2016, 7, 5568-5572.

(23) Berdugo, C.; Miravet, J. F.; Escuder, B. Substrate Selective Catalytic Molecular Hydrogels: The Role of the Hydrophobic Effect. Chem. Commun. 2013, 49, 10608-10610.

(24) Rodríguez-Llansola, F.; Miravet, J. F.; Escuder, B. A Supramolecular Hydrogel as a Reusable Heterogeneous Catalyst for the Direct Aldol Reaction. Chem. Commun. 2009, 7303-7305.

(25) Liu, Y.-R.; He, L.; Zhang, J.; Wang, X.; Su, C.-Y. Evolution of Spherical Assemblies to Fibrous Networked Pd(II) Metallogels from a Pyridine-Based Tripodal Ligand and Their Catalytic Property. Chem. Mater. 2009, 21, 557-563.

(26) Zhang, J.; Wang, X.; He, L.; Chen, L.; Su, C.-Y.; James, S. L. Metal-organic gels as functionalisable supports for catalysis. New J. Chem. 2009, 33, 1070.

(27) Lee, Y.-R.; Kim, J.; Ahn, W.-S. Synthesis of Metal-Organic Frameworks: A Mini Review. Korean J. Chem. Eng. 2013, 30, 16671680.

(28) Malviya, N.; Sonkar, C.; Kundu, B. K.; Mukhopadhyay, S. Discotic Organic Gelators in Ion Sensing, Metallogel Formation, and Bioinspired Catalysis. Langmuir 2018, 34, 11575-11585.

(29) Zhang, W.; Dynes, J. J.; Hu, Y.; Jiang, P.; Ma, S. Porous MetalMetalloporphyrin Gel as Catalytic Binding Pocket for Highly Efficient Synergistic Catalysis. Nat. Commun. 2019, 10, 1913.

(30) Araújo, M.; Muñoz Capdevila, I.; Díaz-Oltra, S.; Escuder, B. Tandem Catalysis of an Aldol-'Click' Reaction System within a Molecular Hydrogel. Molecules 2016, 21, 744.

(31) Liao, Y.; He, L.; Huang, J.; Zhang, J.; Zhuang, L.; Shen, H.; Su, C.-Y. Magnetite Nanoparticle-Supported Coordination Polymer 
Nanofibers: Synthesis and Catalytic Application in Suzuki-Miyaura Coupling. ACS Appl. Mater. Interfaces 2010, 2, 2333-2338.

(32) Tu, T.; Assenmacher, W.; Peterlik, H.; Schnakenburg, G.; Dötz, K. H. Pyridine-Bridged Benzimidazolium Salts: Synthesis, Aggregation, and Application as Phase-Transfer Catalysts. Angew. Chem., Int. Ed. 2008, 47, 7127-7131.

(33) Wang, Q.; Yang, Z.; Wang, L.; Ma, M.; Xu, B. Molecular Hydrogel-Immobilized Enzymes Exhibit Superactivity and High Stability in Organic Solvents. Chem. Commun. 2007, 1032-1034.

(34) Fang, W.; Zhang, Y.; Wu, J.; Liu, C.; Zhu, H.; Tu, T. Recent Advances in Supramolecular Gels and Catalysis. Chem.-Asian J. 2018, 13, 712-729.

(35) Wang, G.; Wang, D.; Bietsch, J.; Chen, A.; Sharma, P. Synthesis of Dendritic Glycoclusters and Their Applications for Supramolecular Gelation and Catalysis. J. Org. Chem. 2020, 85, 16136-16156.

(36) Ta, L.; Axelsson, A.; Bijl, J.; Haukka, M.; Sundén, H. Ionic Liquids as Precatalysts in the Highly Stereoselective Conjugate Addition of $\alpha, \beta$-Unsaturated Aldehydes to Chalcones. Chem.-Eur. J. 2014, 20, 13889-13893.

(37) Axelsson, A.; Ta, L.; Sundén, H. Ionic Liquids as Carbene Catalyst Precursors in the One-Pot Four-Component Assembly of Oxo Triphenylhexanoates (OTHOs). Catalysts 2015, 5, 2052-2067.

(38) Sauvée, C.; Ström, A.; Haukka, M.; Sundén, H. A MultiComponent Reaction towards the Development of Highly Modular Hydrogelators. Chem.-Eur. J. 2018, 24, 8071-8075.

(39) Hsu, C.-W.; Sauvée, C.; Sundén, H.; Andréasson, J. Writing and Erasing Multicolored Information in Diarylethene-Based Supramolecular Gels. Chem. Sci. 2018, 9, 8019-8023.

(40) Johnstone, M. D.; Hsu, C.-W.; Hochbaum, N.; Andréasson, J.; Sundén, H. Multi-Color Emission with Orthogonal Input Triggers from a Diarylethene Pyrene-OTHO Organogelator Cocktail. Chem. Commun. 2020, 56, 988-991.

(41) Baylis, A. B.; Hillman, M. E. D. Offenlegungsschrift. German Patent EP2155113A1, 1972.

(42) Morita, K.-i.; Suzuki, Z.; Hirose, H. A Tertiary Phosphinecatalyzed Reaction of Acrylic Compounds with Aldehydes. Bull. Chem. Soc. Jpn. 1968, 41, 2815.

(43) Wei, Y.; Shi, M. Recent Advances in Organocatalytic Asymmetric Morita-Baylis-Hillman/aza-Morita-Baylis-Hillman Reactions. Chem. Rev. 2013, 113, 6659-6690.

(44) Knoevenegel, E. Condensation von Malondiure Mit Aromatiachen Aldehyden Durch Ammoniak Und Amine. Ber. Dtsch. Chem. Ges. 1898, 31, 2569-2619.

(45) Howe, R. C. T.; Smalley, A. P.; Guttenplan, A. P. M.; Doggett, M. W. R.; Eddleston, M. D.; Tan, J. C.; Lloyd, G. O. A Family of Simple Benzene 1,3,5-Tricarboxamide (BTA) Aromatic Carboxylic Acid Hydrogels. Chem. Commun. 2013, 49, 4268-4270.

(46) Wang, C.; Chen, Q.; Sun, F.; Zhang, D.; Zhang, G.; Huang, Y.; Zhao, R.; Zhu, D. Multistimuli Responsive Organogels Based on a New Gelator Featuring Tetrathiafulvalene and Azobenzene Groups: Reversible Tuning of the Gel-Sol Transition by Redox Reactions and Light Irradiation. J. Am. Chem. Soc. 2010, 132, 3092-3096.

(47) Yang, Z.; Gu, H.; Fu, D.; Gao, P.; Lam, J. K.; Xu, B. Enzymatic Formation of Supramolecular Hydrogels. Adv. Mater. 2004, 16, $1440-1444$.

(48) Jiang, Y.; Zeng, F.; Gong, R.; Guo, Z.; Chen, C.-F.; Wan, X. A multi-stimuli responsive organogel based on a tetrapeptide-dithienylcyclopentene conjugate. Soft Matter 2013, 9, 7538-7544.

(49) Himabindu, M.; Palanisamy, A. Ultrasound- and TemperatureInduced Gelation of Gluconosemicarbazide Gelator in DMSO and Water Mixtures. Gels 2017, 3, 12.

(50) Núñez-Villanueva, D.; Jinks, M. A.; Gómez Magenti, J.; Hunter, C. A. Ultrasound-Induced Gelation of a Giant Macrocycle. Chem. Commun. 2018, 54, 10874-10877.

(51) Jones, C. D.; Steed, J. W. Gels with Sense: Supramolecular Materials That Respond to Heat, Light and Sound. Chem. Soc. Rev. 2016, 45, 6546-6596.
(52) Cravotto, G.; Cintas, P. Molecular Self-Assembly and Patterning Induced by Sound Waves. the Case of Gelation. Chem. Soc. Rev. 2009, 38, 2684-2697.

(53) Paulusse, J. M. J.; Sijbesma, R. P. Molecule-Based Rheology Switching. Angew. Chem., Int. Ed. 2006, 45, 2334-2337.

(54) Bardelang, D. Ultrasound Induced Gelation: A Paradigm Shift. Soft Matter 2009, 5, 1969-1971.

(55) Yu, X.; Chen, L.; Zhang, M.; Yi, T. Low-Molecular-Mass Gels Responding to Ultrasound and Mechanical Stress: Towards SelfHealing Materials. Chem. Soc. Rev. 2014, 43, 5346-5371.

(56) Steed, J. W.; Atwood, J. L. Supramolecular Chemistry, 2nd ed.; John Wiley \& Sons, Ltd: Wiltshire, 2009; pp 888-893.

(57) Diehn, K. K.; Oh, H.; Hashemipour, R.; Weiss, R. G.; Raghavan, S. R. Insights into Organogelation and Its Kinetics from Hansen Solubility Parameters. Toward a Priori Predictions of Molecular Gelation. Soft Matter 2014, 10, 2632-2640.

(58) Nechyporchuk, O.; Belgacem, M. N.; Pignon, F. Current Progress in Rheology of Cellulose Nanofibril Suspensions. Biomacromolecules 2016, 17, 2311-2320.

(59) Dawn, A.; Kumari, H. Low Molecular Weight Supramolecular Gels Under Shear: Rheology as the Tool for Elucidating StructureFunction Correlation. Chem.-Eur. J. 2018, 24, 762-776.

(60) Sahlin, K.; Forsgren, L.; Moberg, T.; Bernin, D.; Rigdahl, M.; Westman, G. Surface Treatment of Cellulose Nanocrystals (CNC): Effects on Dispersion Rheology. Cellulose 2018, 25, 331-345.

(61) Piepenbrock, M.-O. M.; Lloyd, G. O.; Clarke, N.; Steed, J. W. Metal- and Anion-Binding Supramolecular Gels. Chem. Rev. 2010, 110, 1960-2004.

(62) Draper, E. R.; Adams, D. J. Low-Molecular-Weight Gels: The State of the Art. Chem 2017, 3, 390-410.

(63) Dong, X.; Hui, Y.; Xie, S.; Zhang, P.; Zhou, G.; Xie, Z. Schiff Base Supported MCM-41 Catalyzed the Knoevenagel Condensation in Water. RSC Adv. 2013, 3, 3222-3226.

(64) Ding, L.; Li, H.; Zhang, Y.; Zhang, K.; Yuan, H.; Wu, Q.; Zhao, Y.; Jiao, Q.; Shi, D. Basic Polymerized Imidazolide-Based Ionic Liquid: An Efficient Catalyst for Aqueous Knoevenagel Condensation. RSC Adv. 2015, 5, 21415-21421. 